\title{
Indicações de rinosseptoplastia: uma revisão integrativa
}

\author{
Rhinoseptoplasty indications: an integrative review
}

Indicaciones de rinoseptoplastia: una revisión integrativa

Jemima Ferreira Coelho ${ }^{1 *}$, Indiara Iris de Oliveira Araujo1, Laura Pires Ligeiro ${ }^{1}$, Matheus Hybner Gonçalves ${ }^{1}$, Ana Silvia Menezes Bastos' ${ }^{1}$, Riquelme Romero Leal Portela1.

\section{RESUMO}

Objetivo: Abordar sobre a indicação de correção cirúrgica do septo nasal tanto na criança quanto no adulto se atentando à particularidade de cada uma das fases. Métodos: Esta revisão integrativa de literatura especializada teve como base um levantamento bibliográfico acerca das indicações de rinosseptoplastia. As etapas de construção dessa revisão foram: selecionar a temática e a questão de pesquisa, buscar artigos publicados entre 2010 e 2021 nas bases de dados digitais, estabelecer critérios de inclusão, exclusão, e discussão dos artigos, sendo selecionados ao final 28 artigos. Resultados: A rinosseptoplastia é um procedimento que permite a restauração funcional e estética do nariz, sendo então imprescindível avaliar bem o paciente por meio exames complementares e analisar fatores como: a idade do paciente, o tipo de desvio de septo, o grau do desvio, a quantificar da obstrução nasal e presença de outras alterações anatômicas que também necessitem de intervenção cirúrgica, antes de autorizar a operação. Considerações finais: A rinosseptoplastia é um procedimento cujo os resultados dependem da boa indicação cirúrgica, da técnica do cirurgião, e da boa relação médico paciente para que o objetivo da intervenção possa ser alcançado.

Palavras-chave: Rinoplastia, Septo nasal, Obstrução nasal.

\begin{abstract}
Objective: To address the indication for surgical correction of the nasal septum in both children and adults, paying attention to the particularities of each of the phases. Methods: This integrative review of specialized literature was based on a literature review on the indications for rhinoseptoplasty. The stages of construction of this review were: selecting the theme and research question, searching for articles published between 2010 and 2021 in digital databases, establishing criteria for inclusion, exclusion, and discussion of articles, with 28 articles being selected at the end. Results: The rhinoseptoplasty is a procedure that allows the functional and aesthetic restoration of the nose, and it is therefore essential to evaluate the patient well through complementary exams and analyze factors such as: the patient's age, the type of septum deviation, the degree of deviation, to quantify nasal obstruction and the presence of other anatomical changes that also require surgical intervention, before authorizing the operation. Final considerations: The rhinoseptoplasty is a procedure whose results depend on the good surgical indication, the surgeon's technique, and the good doctorpatient relationship so that the intervention objective can be achieved.
\end{abstract}

Keywords: Rhinoplasty, Nasal septum, Nasal obstruction.

\section{RESUMEN}

Objetivo: Abordar la indicación de corrección quirúrgica del tabique nasal tanto en niños como en adultos, prestando atención a la particularidad de cada una de las fases. Métodos: Esta revisión integradora de la literatura especializada se basó en una revisión de la literatura sobre las indicaciones de la rinoseptoplastia.

${ }^{1}$ Universidade de Vassouras (UV), Vassouras - RJ. *E-mail: mima-ferreira@hotmail.com 
Las etapas de construcción de esta revisión fueron: selección del tema y pregunta de investigación, búsqueda de artículos publicados entre 2010 y 2021 en bases de datos digitales, establecimiento de criterios de inclusión, exclusión y discusión de artículos, seleccionándose 28 artículos al final. Resultados: La rinoplastia es un procedimiento que permite la restauración funcional y estética de la nariz, por lo que es fundamental evaluar bien al paciente mediante pruebas complementarias y analizar factores como: la edad del paciente, el tipo de desviación del tabique, el grado de desviació, para cuantificar la obstrucción nasal y la presencia de otros cambios anatómicos que también requieran intervención quirúrgica, antes de autorizar la operación. Consideraciones finales: La rinoseptoplastia es un procedimiento cuyos resultados dependen de la buena indicación quirúrgica, la técnica del cirujano y la buena relación médico-paciente para lograr el objetivo de la intervención.

Palabras clave: Rinoplastia, Tabique nasal, Obstrucción nasal.

\section{INTRODUÇÃO}

A cavidade nasal é dividida anatomicamente pelo septo nasal em direita e esquerda pelo, este é uma estrutura osteocartilaginosa que se estende das narinas até as coanas nasais. Dentre as funções do nariz de olfação, respiração, filtração de poeira, aquecimento e umidificação de $90 \%$ do ar inspirado, ele também é o local onde aflui os ductos lacrimonasais e os seios paranasais; participando então da recepção e eliminação de secreções destes (AZIZ T et al., 2014).

O septo nasal, por ser a estrutura central da cavidade nasal, favorece a existência de um fluxo aéreo laminar, de modo que qualquer alteração em sua estrutura e ou conformação pode levar a diferentes graus de obstrução nasal (MACHADO AM, et al., 2017; TEIXEIRA J, et al., 2016).

A obstrução nasal é a queixa mais comumente relatada pelos pacientes com distúrbios respiratórios, sejam eles crianças ou adultos, impactando de forma negativamente na qualidade de vida destes. Em crianças é muito comum ocorrer o fenômeno de respiração bucal de suplência ao bloqueio nasal (PIRSIG W, 2012).

Esse quadro ao se arrastar durante a fase de crescimento pode levar a distúrbios graves do desenvolvimento físico, sejam faciais, oral, nasal ou até mesmo torácico. Além disso, a criança também pode apresentar prejuízo no desenvolvimento intelectual. No adulto jovem o prejuízo da qualidade de vida é visto na queixa de cansaço ou fadiga constante, bem como intolerância a exercícios físicos e ainda com o aparecimento de roncos que podem ou não ser acompanhados da síndrome da apnéia obstrutiva do sono (MANIGLIA CP e MANIGLIA JV, 2017).

Por se tratar de um sintoma subjetivo antes de se indicar qualquer procedimento e intervenção, a obstrução deve ser amplamente investigada buscando entender a sua etiologia, uma vez que esta pode ser por congestão da mucosa ou por uma alteração anatômica (MACHADO AM, et al., 2017).

A rinosseptoplastia assim como os demais campos da otorrinolaringologia, tem sofrido grandes mudanças na última década, a razão disso é o crescente uso de novos exames de imagem como a fibronasolaringoscopia, bem como o emprego de métodos de minimamente invasivos como as cirurgias endoscópicas. Isso se deve, não somente ao grande anseio estético que a rinosseptoplastia traz consigo para a busca de melhores resultados e redução do índice de complicações como tambem a evolução e aprimoramento das técnicas cirúrgicas já existentes (AZIZ T, et al., 2014; SATHYAKI DC, et al., 2014; SHAFIK AG, et al., 2020).

Isto posto, este trabalho objetiva discorrer sobre as possíveis indicações da rinosseptoplastia e a importância de uma indicação precisa para o desfecho do procedimento pois, o exame pré-operatório que prediz a cirurgia, além de possibilitar o planejamento individualmente as possíveis técnicas que possam ser empregadas com base no caso do paciente, permitindo antever os possíveis resultados de forma realista bem como as possíveis complicações. Em suma, trata-se da importância de se ter um rigor na avaliação préoperatória e de se ter uma precisa indicação cirúrgica a fim de se obter o sucesso da rinosseptoplastia. 


\section{MÉTODOS}

O presente artigo trata-se de um estudo fundamentado em uma revisão integrativa de literatura baseada em evidências objetivando a construção de conhecimento alicerçado e uniforme para a realização de uma prática médica de qualidade. As etapas para construir essa revisão foram: identificar o tema e selecionar a questão de pesquisa, buscar os artigos nas bases de dados digitais, análise dos artigos com aplicação de critérios para inclusão e exclusão de estudos e discussão dos resultados apresentados pelos periódicos.

Este artigo foi escrito com base em uma literatura especializada cuja questão norteadora foi: indicações de rinosseptoplastia, a fim de responder o questionamento: "as rinosseptoplastias estão sendo realizadas dentro dos critérios indicativos? ". Foram buscados trabalhos publicados no período de 2010 a 2021 nas bases de dados Google Acadêmico, LILACS em português e no Pubmed e Scielo em inglês.

$\mathrm{Na}$ base de dados Google Acadêmico com as palavras chaves: "indicações de rinosseptoplastia" foram encontrados 271 resultados com a aplicação do filtro temporal de 2010 a 2021. No LILACS foi buscado por "rinosseptoplastia" sendo encontrados 20 artigos publicados na íntegra entre 2010 a 2021; no Scielo com a palavra-chave "rhinoseptoplasty" foram encontrados 16 artigos no período de tempo estipulado; por fim, no Pubmed com a palavra-chave "rhinoseptoplasty" foram encontrados 16 artigos publicados.

Foram incluídos artigos que abordaram as indicações de rinosseptoplastia tanto em adultos quanto em crianças, artigos cuja intervenção cirúrgica tenha sido realizada como forma de restabelecer a funcionalidade anatômica nasal independente da indicação e também artigos que abordavam métodos e parâmetros de avaliação da funcionalidade nasal bem como os resultados pós procedimentos, como evidenciado no fluxograma da (Figura1).

Como critérios de exclusão foram eliminados os artigos que tangenciam a temática, abordando a rinoplastia e/ou rinosseptoplastia apenas com objetivo estético, excluindo a funcionalidade; artigos que os descritores não estavam correlacionados à proposta de pesquisa. De todos os arquivos encontrados nas quatro bases de dados pesquisadas foram excluídas as duplicatas, além disso, todas as referências dos periódicos selecionados foram analisadas por completo a fim de ampliar 0 arcabouço teórico deste artigo, sendo assim utilizadas no total 28 publicações.

Figura 1 - Fluxograma da seleção dos estudos para esta revisão.

GOOGLE
ACADÊMICO
271 ARTIGOS

\begin{tabular}{|c|}
\hline PUBMED \\
16 ARTIGOS \\
ENCONTRADOS \\
\hline
\end{tabular}

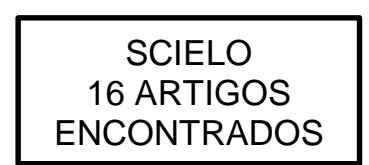

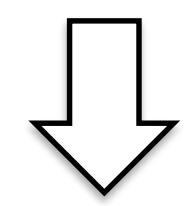

CRITÉRIOS DE INCLUSÃO: artigos que abordaram as indicações de rinosseptoplastia em adultos e/ou crianças, artigos com intervenções cirúrgicas como forma de restabelecer a funcionalidade anatômica nasal, também artigos que abordavam métodos de avaliação da funcionalidade nasal e resultados pós procedimentos.

CRITÉRIOS DE EXCLUSÃO: os artigos que tangenciam a temática, abordando a rinoplastia e/ou rinosseptoplastia apenas com objetivo estético, excluindo a funcionalidade, artigos que os descritores não estavam correlacionados à proposta de pesquisa.

Após aplicação dos critérios citados acima obteve-se um total de 28 artigos.

Fonte: Coelho JF, et al., 2021. 


\section{RESULTADOS}

Após uma leitura cuidadosa das 28 publicações selecionadas, foi feita uma análise crítica e minuciosa do conteúdo de cada uma. Dessa forma, pode-se observar que por se tratar de uma transformação que vem ocorrendo na última década no campo da otorrinolaringologia, ainda são poucos os estudos recentes acerca do emprego dos novos métodos de imagem e tambem dos procedimentos minimamente invasivos como a rinosseptoplastia endoscópica (SATHYAKI DC, et al,.2014; SHAFIK AG, et al,.2020).

Outro ponto contatado, foi que as alterações septo cartilaginosas podem ser vistas em todas as faixas etárias, contudo, como ocorrem diversas mudanças na estrutura óssea da face durante o período de crescimento humano, é mais comum que o diagnóstico de desvio septal seja feito no adolescente e no adulto jovem (MANIGLIA CP e MANIGLIA JV, 2017 e REITZEN SD, et al., 2011).

Outrossim, a rinosseptoplastia é um procedimento cirúrgico muito desafiador para o otorrinolaringologista, seja pela amplitude de técnicas utilizadas, seja pelos resultados que devem ser entregues, já que além de restabelecer a função nasal o procedimento é encarregado de preservar uma estética harmoniosa sempre respeitando a anatomia individual de cada paciente. (ESTEVES SS, et al., 2017).

Por conseguinte, esse procedimento cirúrgico deve ter uma indicação precisa e parcimoniosa. E para indicar com segurança e precisão uma rinosseptoplastia é necessário uma história clínica e exame físico de qualidade para levantar hipóteses diagnósticas compatíveis com obstrução nasal como: desvio septal anterior, hipertrofia de conchas nasais ou rinopatia alérgica. Mas sobretudo, o deve-se questionar sobre a morbidade que essa alteração anatômica traz para o indivíduo, ou seja, qual é a sua correlação com o sintoma queixado seja pelo paciente para que assim possamos avaliar a necessidade de intervenção cirúrgica bem como quais seriam as manobras realizadas nesse procedimento (AZIZ T, et al., 2014; VIAN HNK, et al., 2018).

Durante a avaliação pré-operatória e planejamento do procedimento onde será definida a técnica que será utilizada, além do exame físico intranasal do paciente, os exames complementares também são uma ótima ferramenta e é indispensável ao especialista já que estes ajudam a entender as características da alteração septal bem como seu impacto no quadro da obstrução nasal (AZIZ T, et al., 2014).

Dos exames, o mais simples que temos é a endoscopia nasal, com a qual podemos avaliar toda a cavidade nasal. Também pode ser feita uma rinometria acústica que mensura o volume da cavidade nasal e a área de secção transversa no eixo anteroposterior (passagem do ar), ou uma rinomanometria que mensura a resistência nasal ao fluxo aerífero sendo este o exame eleito para pacientes com queixa de obstrução nasal. Com relação a exames de imagem temos a tomografia computadorizada que permite avaliar com precisão a anatomia do septo e suas relações com a cavidade nasal (MACHADO AM, et al., 2017; PINNA FR e BENTO RF. 2018).

Nos artigos coletados foi visto que as informações obtidas nestes se somam e pouco se divergem, por isso foi possível, levando em conta os dados coletados em cada periódico, dispor em uma tabela os dados cruciais para responder o questionamento proposto. Segue abaixo o Quadro 1 para comparação dos dados coletados a título de comparação e exemplificado de tudo que foi dito até então: 
Quadro 1 - Resultados obtidos após análise dos periódicos.

\begin{tabular}{|c|c|c|c|c|}
\hline TíTULO & $\begin{array}{l}\text { AUTOR/ ANO DE } \\
\text { PUBLICAÇÃO }\end{array}$ & PERFIL EPIDEMIOLÓGICO & INDICAÇÃO CIRÚRGICA & COMPLICAÇÕES \\
\hline $\begin{array}{llr}\text { Pediatric } & \text { Rhinoplasty } & \text { Treatment } \\
\text { Outcomes: } & \text { Asian Medical Center } \\
\text { Experience. } & & \\
\end{array}$ & BAE JS, et al., 2013. & $\begin{array}{l}\text { Estudo consistiu de } 64 \text { crianças coreanas entre } 4 \\
\text { e } 17 \text { anos de idade que foram submetidas a } \\
\text { rinoplastia entre maio de } 2003 \text { e agosto de } 2011 \text {. }\end{array}$ & $\begin{array}{l}\text { Nariz torto, fratura e nariz } \\
\text { achatado. }\end{array}$ & - \\
\hline $\begin{array}{l}\text { Justification for Rhinoseptoplasty in } \\
\text { Children - Our } 10 \text { Years Overview }\end{array}$ & $\begin{array}{l}\text { BARSOVA GK, } \\
\text { NIKOLOVSKI N, } 2016 .\end{array}$ & $\begin{array}{l}\text { Avaliou } 97 \text { crianças de } 6 \text { a } 14 \text { anos, com graves } \\
\text { deformidades nasais e problemas respiratórios } \\
\text { pelo nariz, foram internados para rinoplastia de } \\
2006 \text { a } 2016\end{array}$ & Deformidades no nariz & - \\
\hline $\begin{array}{l}\text { Análise Retrospectiva de Cirurgias } \\
\text { Rinossinusais em um Hospital Escola. }\end{array}$ & $\begin{array}{l}\text { CARVALHO TBO, et } \\
\text { al., } 2010 .\end{array}$ & $\begin{array}{l}\text { Revisão de } 872 \text { prontuários de pacientes } \\
\text { submetidos a cirurgias rinossinusais entre janeiro } \\
\text { de } 2006 \text { e dezembro de } 2008\end{array}$ & $\begin{array}{l}\text { Desvio de septo e deformidade } \\
\text { nasal pós trauma }\end{array}$ & - \\
\hline $\begin{array}{l}\text { Evaluation of aesthetic and functional } \\
\text { outcomes in rhinoplasty surgery: a } \\
\text { prospective study. }\end{array}$ & $\begin{array}{l}\text { ESTEVES SS, et al., } \\
2017 .\end{array}$ & $\begin{array}{l}\text { Estudo prospectivo com todas as cirurgias de } \\
\text { rinoplastia realizadas em adultos entre fevereiro } \\
\text { de } 2013 \text { e agosto de } 2014\end{array}$ & Questões funcionais. & - \\
\hline $\begin{array}{l}\text { Revisão bibliográfica e planejamento de } \\
\text { protocolo através de questionário } \\
\text { sinonasal outcome test, rinometria e } \\
\text { tomografia computadorizada em } \\
\text { doentes propostos para septoplastia. }\end{array}$ & $\begin{array}{l}\text { MACHADO AM, et al., } \\
2017 .\end{array}$ & $\begin{array}{l}\text { Analisados os doentes indicados a septoplastia } \\
\text { no centro hospitalar e universitário de coimbra }\end{array}$ & $\begin{array}{l}\text { Queixas do doente e avaliação } \\
\text { clínica. }\end{array}$ & ( \\
\hline Rhinoseptoplasty in children & $\begin{array}{l}\text { MANIGLIA CP, } \\
\text { MANIGLIA JV, } 2017 .\end{array}$ & $\begin{array}{l}\text { Avaliados } 2022 \text { prontuários de crianças de } 1994 \\
\text { a } 2010\end{array}$ & Alterações congênitas & $\begin{array}{l}\text { Persistência ou recorrência do } \\
\text { desvio, desvio da pirâmide nasal } \\
\text { com deformidade estética, } \\
\text { sangramento, } \\
\begin{array}{l}\text { hematoma septal, abscesão, } \\
\text { septal, sinéquia, perfuração }\end{array} \\
\begin{array}{l}\text { septal, anestesia palatal e } \\
\text { dental. }\end{array}\end{array}$ \\
\hline $\begin{array}{l}\text { Classification of rhinoplasties performed } \\
\text { in an otorhinolaryngology referral center } \\
\text { in Brazil. }\end{array}$ & NUNES FB, et al., 2014. & $\begin{array}{l}\text { Analisados } 325 \text { rinoplastias realizados em um } \\
\text { hospital de janeiro de } 2003 \text { a agosto de } 2012\end{array}$ & $\begin{array}{l}\text { Lesões traumáticas, congênitas } \\
\text { ou de desenvolvimento }\end{array}$ & - \\
\hline
\end{tabular}




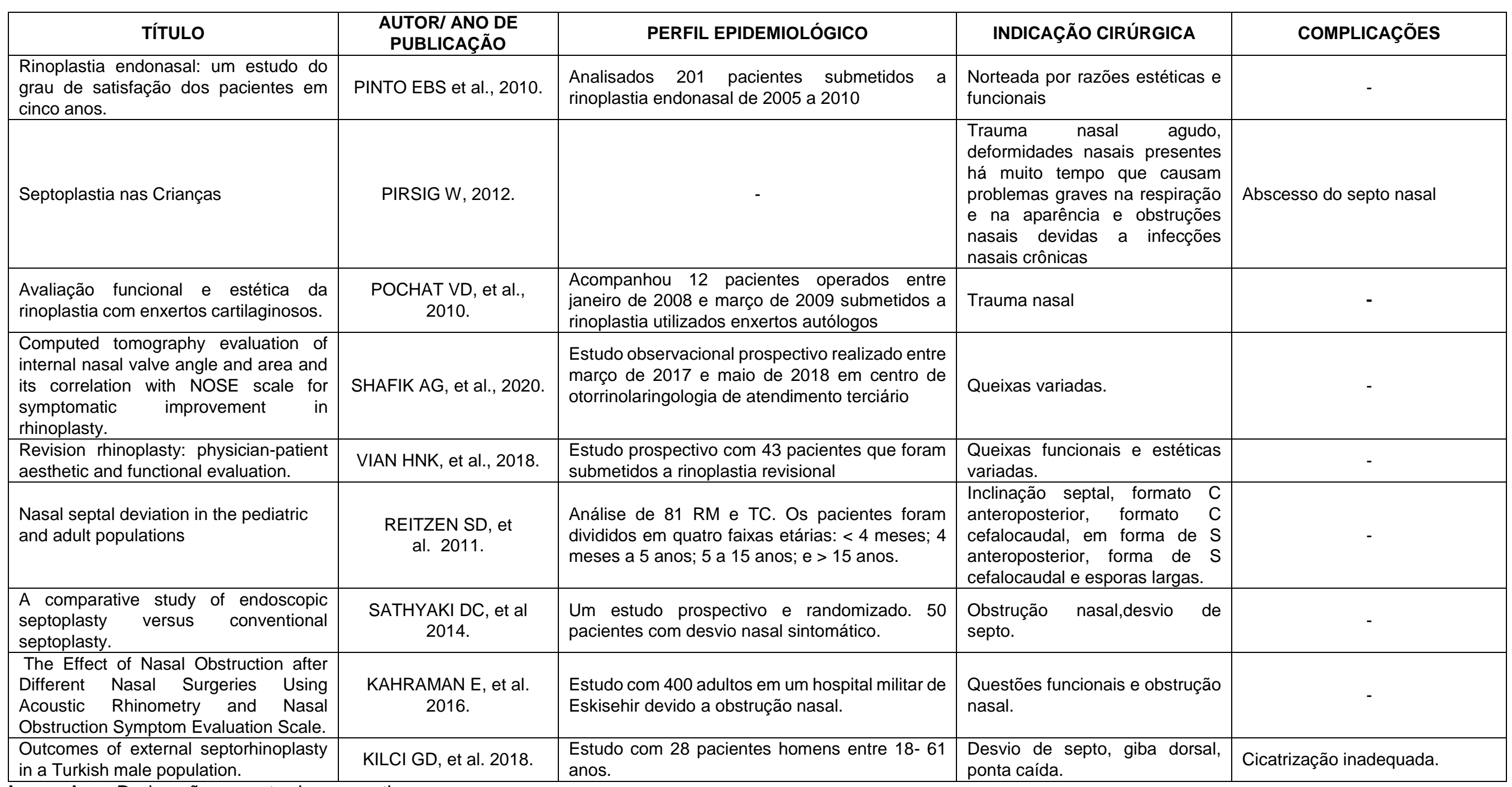

Legenda: -: Dados não encontrados nos artigos.

Fonte: Coelho JF, et al., 2021. 


\section{DISCUSSÃO}

Diante de tudo que foi exposto até o momento, é possível inferir que dentre todos os fatores que devem ser levados em conta ao se indicar o procedimento, a idade do paciente é um fator importante a se considerar. Diversos trabalhos apontam que a rinosseptoplastia deve ser pensada com parcimônia em crianças e adolescentes com menos de 15 anos de idade, devendo esta ter uma indicação ser muito precisa, principalmente se realizada na primeira década de vida. Essa cautela se deve ao fato de o desenvolvimento e crescimento facial estarem incompletos (MANIGLIA CP e MANIGLIA JV, 2017 e REITZEN SD, et al., 2011).

O fato do nariz traumatizado pela cirurgia estar sujeito a mudanças devido a alterações fisiológicas e intrínsecas do próprio desenvolvimento humano tais como: mudança no padrão da respiração, a troca de dentição, o desenvolvimento cerebral, amadurecimento da própria musculatura facial e faríngea; realizar intervenções de forma muito precoce podem levar a outras patologias da cartilagem como por exemplo o desvio septo lateral (MANIGLIA CP e MANIGLIA JV, 2017; PIRSIG W, 2012; REITZEN SD, et al.,2011). Isso foi mostrado em um estudo realizado em um serviço de otorrinolaringologia pediátrica onde foram analisadas 202 crianças submetidas à rinosseptoplastia, e as complicações cirúrgicas mais encontradas foram: recidiva/persistência do desvio septal em 14\%, desvio da pirâmide nasal $4 \%$ e sinéquias $2 \%$ (MANIGLIA CP e MANIGLIA JV, 2017).

Outro ponto a ser analisado tanto em adultos quanto na população pediátrica é a presença de outras disfunções otorrinolaringológicas associadas ao desvio septal que também tenham indicação cirúrgica tais quais: a cirurgia de conchas nasais, a adenoidectomia, e amigdalectomia ou até mesmo microcirurgia otológica. Nesse caso tem sido recomendado que se realize essas intervenções no mesmo ato cirúrgico, ou seja, junto com a rinosseptoplastia (MANIGLIA CP e MANIGLIA JV, 2017).

Ainda na abordagem pré-operatória é indispensável que o profissional consiga explicar ao paciente de forma mais clara e objetiva possível sobre a intervenção a ser realizada, mostrando a intervenção cirúrgica como meio para se restabelecer a função respiratória nasal e também para que o paciente não crie expectativas estéticas utópicas com relação ao procedimento independente da sua indicação. Em um estudo realizado de caráter prospectivo sobre os desfechos estéticos e funcionais de rinoplastia, onde foi feito um acompanhamento em 3 e 6 meses de pós-operatório com 107 pacientes mostrou que, os próprios pacientes relataram melhora significativa da função respiratória e da qualidade de vida, bem como, satisfação com o resultado estético; dos 107 pacientes cerca de $72,9 \%$ responderam que "com certeza" fariam a cirurgia novamente depois de conhecerem os finais (ESTEVES SS, et al., 2016).

Esse estudo também apontou que um grande entrave para a compreensão do paciente acerca do procedimento cirúrgico é o seu grau de alfabetização; com esses dados pode-se então correlacionar o grau de satisfação do pós operatório com o índice de escolaridade do paciente e nele foi visto que os pacientes com menor grau de alfabetização estavam mais satisfeitos com o procedimento em detrimento dos pacientes com maior grau de instrução acadêmica, isso se deve ao fato de que estes pacientes com menor escolaridade tendem a terem menor expectativa com relação ao procedimento consequentemente se sentem mais satisfeitos com os resultados obtidos (ESTEVES SS, et al., 2017).

Em outro estudo também sobre grau de satisfação dos pacientes após cinco anos da cirurgia, foi constatado que a maioria dos participantes operados buscou o procedimento tendo como principal finalidade a melhora estética; nesse estudo, dos 201 pacientes entrevistados cerca de $83,87 \%$ dos indivíduos relataram melhora no padrão respiratório após o procedimento, sendo que destes apenas $6,45 \%$ indivíduos haviam se queixado de obstrução nasal antes do procedimento. Isso mostra que, mesmo os pacientes que não reconheciam a obstrução nasal como queixa principal e que buscaram realizar o procedimento apenas com objetivo estético acabaram por obter uma melhora da função respiratória, demonstrando que a rinoplastia por si só também apresenta um ótimo resultado funcional (PINTO EBS, et al., 2010).

Sob o ponto de vista anatômico a estrutura septal não é plana em toda a sua extensão, ele possui duas saliências, sendo uma delas na altura do corpo cavernoso e outro próximo a crista maxilar. Sendo assim, deformidades mesmo que pequenas na região septo nasal pode gerar variados graus de obstrução nasal. As 
alterações anatômicas septais podem ser classificadas pelos tipos de desvios e pelos graus de desvios encontrados. Com relação aos tipos desvios de septo tem seis tipos: desvio em "C", desvio em "S", desvio caudal, desvio da crista, projeção em esporão e desvio misto (REITZEN SD, et al., 2011).

Já com relação ao grau de desvio são três tipos: sendo o desvio de $1^{\circ}$ grau uma deflexão entre 0 e $33 \%$ em relação à linha média e não chega a tocar a concha inferior. O desvio de $2^{\circ}$ grau é uma deflexão entre $34 \%$ e $66 \%$ em relação à linha média, chegando a tocar a concha inferior. Já o desvio 3o grau uma deflexão entre 67 e 100\% em relação à linha média por isso aqui o septo comprime a concha inferior (TEIXEIRA J, et al., 2018).

À vista disso, entende-se que a rinosseptoplastia é um procedimento cirúrgico que contempla duas modalidades em um procedimento, sendo elas a rinoplastia e a septoplastia. É importante ressaltar que a rinoplastia pode ser executada de forma independente da septoplastia tal qual, por vezes, a septoplastia será a própria indicação de intervenção da rinoplastia, como no caso de uma obstrução nasal ipsilateral à deformidade septal, também no caso de recorrentes epistaxes causadas pela deformidade septal ou ainda uma deformidade septal tamanha que prejudique a execução de outras cirurgias como da pirâmide nasal e procedimentos que porventura venham usar a cavidade nasal como via de acesso (AZIZ T, et al., 2014; JUNIOR NA, et al., 2019; TEIXEIRA J, et al., 2018; SATHYAKI DC, et al., 2014).

No que tange a escolha da técnica para a abordagem cirúrgica na septoplastia, deve ter como prioridade a resolução das queixas relatadas pelo paciente bem como as alterações anatômicas encontradas e dimensionadas nos exames de imagem. (SATHYAKI DC, et al., 2014). Quando se trata de desvios e esporões na região do átrio nasal ou na região das conchas nasais anterior e posterior pode-se utilizar tanto a técnica de Killian \& Freer que introduziram a dissecção subpericondral e subperiosteal, quanto a técnica de Cottle, Guillain onde é feito túneis de dissecção (TAUB PJ e BAKER SB, 2013).

A técnica de Metzenbaum onde é feita uma incisão vertical ao longo do vértice do desvio septal, permite uma boa exposição do septo caudal, sendo assim, é mais utilizado em desvios de região caudal. Já o vídeo endoscópio se mostra uma boa opção na abordagem de esporões em região anterior e posterior das conchas nasais, pois permite uma abordagem direta do esporão com excelente campo de visão, não necessitando então realizar dissecções em áreas distantes (JUNIOR NA, et al., 2019; SATHYAKI DC, et al., 2014).

Para os desvios complexos, onde pode haver necessidade de retirada em bloco da cartilagem quadrangular, condroplastia ou reintrodução com fixação da cartilagem na cavidade nasal é necessário fazer uso do procedimento extracorpóreo. Eventualmente, devido a ocorrência de variações anatômicas que conferem características únicas a determinadas alterações do septo, para esses pacientes será necessário utilizar passos cirúrgicos de diversas técnicas e em uma mesma intervenção, sendo assim o procedimento é denominado de técnica mista (JUNIOR NA, et al., 2019; SATHYAKI DC, et al., 2014).

Isto posto, consegue-se compreender que a rinosseptoplastia funcional é realizada quando há alterações do septo nasal em concomitância a deformidades da pirâmide nasal, vestíbulo nasal ou válvula nasal, e para isso é necessária uma exposição dos elementos anatômicos da pirâmide nasal de tal forma que permita sua manipulação (ÇAĞıCI CA, 2017; JUNIOR NA, et al., 2018).

No que se refere a complicações do procedimento de rinosseptoplastia é possível compreender que as complicações variam conforme a técnica escolhida para abordagem e a região mais manipulada, motivo pelo qual algumas regiões do septo nasal devem ser poupadas ou quando abordadas deve ser feita com máximo de cautela possível a fim de evitar complicações graves. A dor pós-operatória em rinosseptoplastia é tida como preditivo de complicações, tais quais: fístula liquórica, infecção do sítio cirúrgico, abscesso, hematoma, seroma, impactação do split na parede nasal ou ainda isquemia do tecido decorrente da sutura do splint (VIAN HNK, et al., 2018; GOMES OS, et al.,2021; BAE JS, et al., 2013).

Ainda ponderando sobre as complicações pós-operatórias em rinosseptoplastia, das informações colhidas nos artigos levantados, a título de comparação foram correlacionados algumas complicações e as suas causas em uma tabela (Quadro 2). 
Quadro 2 - Correlação entre complicações, suas causas e áreas de ocorrência.

\begin{tabular}{|c|c|c|}
\hline COMPLICAÇŌES & ÁREA AFETADA & CAUSAS \\
\hline Desvio iatrogênico & Toda a região septal & Tamponamento unilateral \\
\hline Epistaxe & $\begin{array}{l}\text { Limite posterior do septo nasal próximo a } \\
\text { artéria septal posterior. }\end{array}$ & $\begin{array}{l}\text { Ressecção septal próximo ao osso esfenóide } \\
\text { ou uso de splint nasal muito posterior. }\end{array}$ \\
\hline $\begin{array}{l}\text { Coleção, Seroma e } \\
\text { Abscesso. }\end{array}$ & Região intraseptal & $\begin{array}{l}\text { Não realização da incisão unilateral na } \\
\text { mucosa para facilitar a drenagem. }\end{array}$ \\
\hline Infecção & Todo o sítio cirúrgico & $\begin{array}{l}\text { Tamponamento nasal sem antibioticoprofilaxia } \\
\text { adequada. }\end{array}$ \\
\hline Fístula liquórica & $\begin{array}{l}\text { Junção do terço médio da lâmina } \\
\text { perpendicular do etmóide com teto nasal }\end{array}$ & $\begin{array}{l}\text { Uso de escopro na lâmina perpendicular do } \\
\text { etmóide, levando a fratura de base de crânio e } \\
\text { consequentemente a fistulação. }\end{array}$ \\
\hline Ptose de ponta & $\begin{array}{l}\text { Borda anterior e superior da cartilagem } \\
\text { quadrangular. }\end{array}$ & $\begin{array}{l}\text { Não preservação do "L" estrutural ou } \\
\text { reconstrução deste de forma inadequada. }\end{array}$ \\
\hline Nariz em sela & $\begin{array}{l}\text { Area de junção entre a lâmina perpendicular } \\
\text { do etmóide, cartilagem lateral superior, } \\
\text { cartilagem quadrangular e osso próprio do } \\
\text { nariz. }\end{array}$ & $\begin{array}{l}\text { Não fixação da Zona "K" com suturas deixando } \\
\text { desarticulações. }\end{array}$ \\
\hline
\end{tabular}

Fonte: Coelho JF, et al., 2021.

Ainda que seja notório o risco que advém com a realização de intervenção cirurgia em uma área que está em desenvolvimento, a rinosseptoplastia em crianças têm se mostrado extremamente benéfica, por restabelecer a permeabilidade das fossas nasais permitindo um crescimento adequado do complexo nasomaxilar, quando se comparado com as consequências prejudiciais ocasionados pela falta da intervenção como as sequelas pela respiração bucal ao longo dos anos (PINTO EBS, et al., 2010; LAWRENCE R, 2012; REITZEN SD, et al., 2011). Dessa forma, em pacientes pediátricos tem se optado por intervenções mais conservadoras em que se atenda a necessidade de melhorar a qualidade da respiração da criança sem comprometer a estética dela durante o crescimento fisiológico (PINTO EBS, et al., 2010; PIRSIG W, 2012).

No decorrer da avaliação e preparação para a cirurgia é necessário abordar com o paciente todos os aspectos do procedimento, desde os resultados esperado, as possíveis complicações e até mesmo a possibilidade de que com os resultados do procedimento haver necessidade de retoques ou reoperações para corrigir mais os desvios (MANIGLIA CP e MANIGLIA JV, 2017). Mesmo o ato cirúrgico tendo o intuito primordial de restabelecer a função nasal, durante o planejamento de conduta para 0 ato cirúrgico não podemos deixar de atender também às expectativas estéticas do paciente, desde que estas sejam realistas, devendo-se sempre individualizar cada caso (PINTO EBS, et al., 2010).

A rinosseptoplastia é capaz de melhorar significativamente a qualidade de vida do paciente, tanto na retomada de sua função respiratória quanto na obtenção de resultados cosméticos favoráveis, visto que mesmo os pacientes que não reconhecem a obstrução nasal como queixa motivadora da cirurgia no pósoperatório relatam melhora da respiração. Esse achado certamente pode ser atribuído ao fato de que ao longo dos anos esses pacientes que possuíam qualquer grau de obstrução nasal que não fosse completa encontraram uma forma de se adaptar para compensar a deficiência nasal, seja por meio de uma respiração bucal de suplência ou qualquer outro artifício para manter o fluxo respiratório; estes pacientes quando submetidos a rinoplastia associada a rinodinâmica e ao terem o fluxo laminar nasal restabelecido puderam perceber melhora significativa em sua respiração o que impactou positivamente a sua qualidade de vida a ponto de serem relatados no pós-operatório (PINTO EBS, et al., 2010; VIAN HNK, et al., 2018).

A otorrinolaringologia como especialidade tem crescido e evoluído bastante na última década, e no que tange ao uso de novos exames complementares de imagem para indicações de procedimentos e análise de desfechos pós-operatórios em cirurgias plásticas, ainda é escasso o número de publicações recentes sobre o tema, todavia, foi visto que a padronização da avaliação pré e pós operatória nas rinosseptoplastias permite não somente um desfecho mais favorável para a recuperação da função nasal como a diminuição da incidências de complicações; posto que a maioria das obstruções nasais se deve a desvios da cartilagem septal, mas não é infrequente encontrar outras alterações anatômicas em associação e estas, devem sempre que possível serem realizadas no mesmo procedimento cirúrgico (AZIZ T, et al., 2014; SATHYAKI DC, et al., 2014; SHAFIK AG, et al., 2020). 


\section{CONSIDERAÇÕES FINAIS}

Em suma, é possível depreender dos dados aqui levantados que a manutenção da respiração nasal fisiológica está intimamente relacionada com a qualidade de vida do paciente, e que a rinosseptoplastia é uma cirurgia complexa que exige grande habilidade cirúrgica, por isso é necessário realizar uma avaliação pré-operatória ampla e minuciosa, o que permite uma abordagem mais individualizada do caso do paciente. O sucesso do procedimento depende tanto da técnica abordada pelo cirurgião quanto do grau de entendimento do paciente sobre a cirurgia e nesse ponto uma das peças chaves é cultivar uma boa relação médico paciente onde o profissional seja capaz de passar segurança para o paciente sempre explicando de forma clara e compreensível o procedimento, de forma a atingir o objetivo proposto e evitar que se crie falsas expectativas.

\section{REFERÊNCIAS}

1. AZIZ T, et al,. Measurement tools for the diagnosis of nasal septal deviation: a systematic review. Otolaryngology Head and Neck Surg, 2014;43:11.

2. $\mathrm{BAE}$ JS, et al,. Resultados do tratamento da rinoplastia pediátrica: a experiência do Asian Medical Center. International Journal of Pediatric Otorhinolaryngology, 2013; 77(10): 1701-1710.

3. BARSOVA GK, Nikolovski N. Justification for Rhinoseptoplasty in Children -Our 10 Years Overview. Journal of Medical Sciences, 2016;4 (3): 397-403.

4. ÇAĞICI CA. Evaluation of postoperative patient satisfaction after covering the nasal dorsum with upper lateral cartilage: "upper lateral closing". Braz J Otorhinolaryngol, 2019; 85(1):71-77.

5. CARVALHO TBO, et al,. Análise Retrospectiva de Cirurgias Rinossinusais em um Hospital Escola. Arq. Int. Otorrinolaringol, 2010; 14(4): 428-43.

6. DOLCI EL, DOLCI JEL. Algorithm for the treatment of external nasal valve insufficiency. Braz. J Otorhinolaryngol, 2020; 86(50): 579-586.

7. ESTEVES SS, et al,.. Evaluation of aesthetic and functional outcomes in rhinoplasty surgery: a prospective study. Braz J Otorhinolaryngol, 2017; 83(5):552-557.

8. GOMES OS, et al. Cirurgia plástica no Brasil: uma análise epidemiológica. Revista Eletrônica Acervo Científico, 2021.

9. JUNIOR NA, et al,. Ajuste e reposicionamento da pirâmide e septo nasal - uma técnica conservadora e eficaz em Rinoplastia. Braz. J Otorhinolaryngol, 2019; 85(2): 176-182.

10. KAYABASI $\mathrm{S}$, et al. Os efeitos do tempo de cirurgia intradia na dor e ansiedade de pacientes submetidos à septoplastia. Braz. J Otorhinolaryngol, 2021;87(3):310-314.

11. KAHRAMAN E, et al., The Effect of Nasal Obstruction after Different Nasal Surgeries Using Acoustic Rhinometry and Nasal Obstruction Symptom Evaluation Scale. Word J Plast Surg, 2016; 5 (3): 236-243.

12. KILCI GD, et al. Outcomes of external septorhinoplasty in Turkish male population. Braz. J Otorhinolarongol, 2018; 84(4):426-434

13. LAWRENCE R. Pediatric septoplasy: a review of the literature. International Jour-nal of Pediatric Otorhinolaryngology, 2012;76:1078-81.

14. MACHADO AM, et al,. Revisão bibliográfica e planejamento de protocolo através de questionário sinonasal outcome test, rinometria e tomografia computadorizada em doentes propostos para septoplastia. Revista Portuguesa Otorrinolaringologia e Cirurgia de Cabeça e Pescoço, 2017; 55(4): 183-86.

15. MANIGLIA CP, MANIGLIA JV. Rhinoseptoplasty in children. Braz J Otorhinolaryngol, 2017; 83(4):416-19.

16. MOORE M, ECCLES R. Objective evidence for the efficacy of surgical management of the deviated septum as a treatment for chronic nasal obstruction: a systematic review. Wiley Online Library Clinical Otolaryngological, 2011; 36(2): 106-113.

17. NUNES FB, et al,. Classification of rhinoplasties performed in an otorhinolaryngology referral center in Brazil. Braz J Otorhinolaryngol, 2014; 80(6):476-9.

18. PINTO EBS, et al. Rinoplastia endonasal: um estudo do grau de satisfação dos pacientes em cinco anos. Revista Brasileira de Cirurgia Plástica, 2010; 25(4): 637-40.

19. PIRSIG W. Septoplastia nas crianças. XI Manual de Otorrinolaringologia Pediátrica da IAPO, 2012; 204-212.

20. POCHAT VD, Alonso N, Meneses JVL. Avaliação funcional e estética da rinoplastia com enxertos cartilaginosos. Rev Bras Cir Plást, 2010; 25(2): 260-70.

21. REITZEN SD, et al,. Nasal septal deviation in the pediatric and adult populations. ENT-Ear, Nose \& Throat Journal, 2011; 90(3): 112-115.

22. RESENDE L, et al,. Disease-specific quality of life after septoplasty and bilateral inferior turbinate outfracture in patients with nasal obstruction. Braz J Otorhinolaryngology, 2018; 84(5):591-598.

23. SATHYAKI DC, et al,. A comparative study of endoscopic septoplasty versus conventional septoplasty. Indian J Otolaryngol Head Neck Surg, 2014;66(2):155-61.

24. SAZGAR AA, et al. Diferentes técnicas para colocação de enxerto de extensão causal em rinoplastia. Braz. J Otorhinolaryngol, 2021; 87:188-192.

25. SHAFIK AG, et al,. Computed tomography evaluation of internal nasal valve angle and area and its correlation with NOSE scale for symptomatic improvement in rhinoplasty. Braz J Otorhinolaryngol, 2020;86(3):343-350.

26. TAUB PJ e BAKER SB. Rinoplastia: Série Atlas de Cirurgia Plástica. New York-NY: AMGH Editora Ltda, 2013.

27. TEIXEIRA J, et al,. Nasal septal deviations: a systematic review of classification systems. Surgery International, 2016; e 7089123

28. VIAN HNK, et al,. Revision rhinoplasty: physician-patient aesthetic and functional evaluation. Braz J Otorhinolaryngol. 2018, 84(6):736-743. 